\title{
Proximity-Induced Shiba States in a Molecular Junction
}

\author{
Joshua O. Island, ${ }^{1, *}$ Rocco Gaudenzi, ${ }^{1}$ Joeri de Bruijckere, ${ }^{1}$ Enrique Burzurí, ${ }^{1}$ \\ Carlos Franco, ${ }^{2}$ Marta Mas-Torrent, ${ }^{2}$ Concepció Rovira, ${ }^{2}$ Jaume Veciana, ${ }^{2}$ \\ Teun M. Klapwijk, ${ }^{1,3}$ Ramón Aguado, ${ }^{4}$ and Herre S. J. van der Zant ${ }^{1}$ \\ ${ }^{1}$ Kavli Institute of Nanoscience, Delft University of Technology, Lorentzweg 1, 2628 CJ Delft, Netherlands \\ ${ }^{2}$ Institut de Ciéncia de Materials de Barcelona (ICMAB-CSIC) and CIBER-BBN, \\ Campus de la UAB, 08193 Bellaterra, Spain \\ ${ }^{3}$ Physics Department, Moscow State Pedagogical University, Moscow 119991, Russia \\ ${ }^{4}$ Instituto de Ciencia de Materiales de Madrid, Consejo Superior de Investigaciones Científicas (ICMM-CSIC), \\ Sor Juana Inés de la Cruz 3, 28049 Madrid, Spain \\ (Received 4 October 2016; revised manuscript received 6 January 2017; published 17 March 2017)
}

\begin{abstract}
Superconductors containing magnetic impurities exhibit intriguing phenomena derived from the competition between Cooper pairing and Kondo screening. At the heart of this competition are the Yu-Shiba-Rusinov (Shiba) states which arise from the pair breaking effects a magnetic impurity has on a superconducting host. Hybrid superconductor-molecular junctions offer unique access to these states but the added complexity in fabricating such devices has kept their exploration to a minimum. Here, we report on the successful integration of a model spin $1 / 2$ impurity, in the form of a neutral and stable all organic radical molecule, in proximity-induced superconducting break junctions. Our measurements reveal excitations which are characteristic of a spin-induced Shiba state due to the radical's unpaired spin strongly coupled to a superconductor. By virtue of a variable molecule-electrode coupling, we access both the singlet and doublet ground states of the hybrid system which give rise to the doublet and singlet Shiba excited states, respectively. Our results show that Shiba states are a robust feature of the interaction between a paramagnetic impurity and a proximity-induced superconductor where the excited state is mediated by correlated electron-hole (Andreev) pairs instead of Cooper pairs.
\end{abstract}

DOI: 10.1103/PhysRevLett.118.117001

A quantum dot (QD) or impurity coupled to a superconductor constitutes a rich physical system in which many-body effects compete for the ground state [1-9]. The ground state can take the form of a BCS-like singlet, a spin degenerate doublet, or a Kondo-like singlet, depending on the relative strengths of the characteristic energies of the competing phenomena (charging energy, $U$, superconducting gap, $\Delta$, Kondo energy, $k_{B} T_{K}$ ). For a weak Coulomb interaction $(U \ll \Delta)$, the BCS singlet, composed of the superposition of unoccupied and doubly occupied states of the dot, prevails. Subgap excitations of this ground state are the well-known Andreev bound states. This regime has been explored in carbon nanotube $[10,11]$ and nanowire $[12,13]$ devices where $\Delta$ can be large enough relative to $U$ to allow the BCS superposition state. For larger charging energy, however, the doublet becomes the energetically favored ground state (at temperatures above $T_{K}$ ) and a competition between Kondo screening and Cooper pairing sets in at temperatures below $T_{K}[8,14]$. For weak Kondo energy $\left(k_{B} T_{K} \ll \Delta\right)$, the ground state is the degenerate doublet as screening is incomplete due to a lack of quasiparticles at the Fermi level. For strong Kondo energy $\left(k_{B} T_{K} \gg \Delta\right)$, quasiparticles screen the spin and the Kondo singlet becomes the ground state. Excitations on top of these ground states are the Yu-Shiba-Rusinov (or simply Shiba) states [15-18]. First experimentally observed in tunneling spectra of magnetic adatoms absorbed on $\mathrm{Nb}$ [19], these states were recently shown to lead to topological Shiba bands required for the observation of Majorana end modes in atomic chains adsorbed on a superconducting surface [20-22].

Besides early tunneling experiments on Kondo alloys producing conventional Shiba bands [23-25], the great majority of investigations of superconductor-QD systems are with magnetic impurities [2,7,19,26], nanowires [3,4], or nanotubes [27-29] coupled directly to a bulk superconductor. In these systems, the singlet Shiba excited state is created by breaking a Cooper pair which allows a quasiparticle to pair with the localized spin in the quantized system. A similar interaction may occur through proximity induced superconductivity but has not been explored and requires attention as proposals and investigations of Majorana bound states engineered through proximity induced systems grow [12,30-35].

The hybrid superconductor-molecule device grants a unique exploration of the superconductor-QD phase diagram as the energy spacing between molecular orbitals of a molecule is typically orders of magnitude larger than the other energy scales completely suppressing the formation of the BCS-like singlet ground state and offering investigation of the large- $U$ regime. The added difficulty in fabricating such devices has limited their investigation to only a few studies [36,37]. Winkelmann et al. have achieved contact to $\mathrm{C}_{60}$ molecules using proximity induced gold and aluminum electrodes which showed the interplay of a spin- $1 / 2$ impurity 
(a)

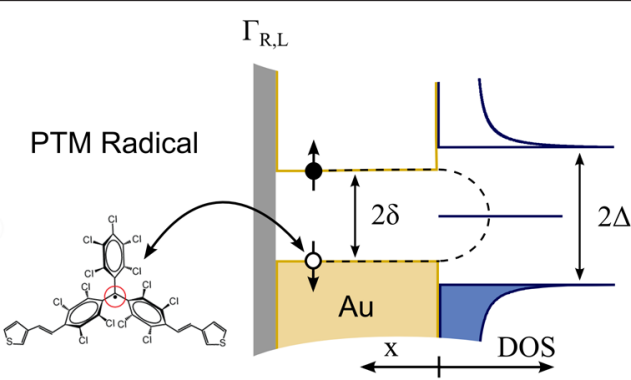

(b)

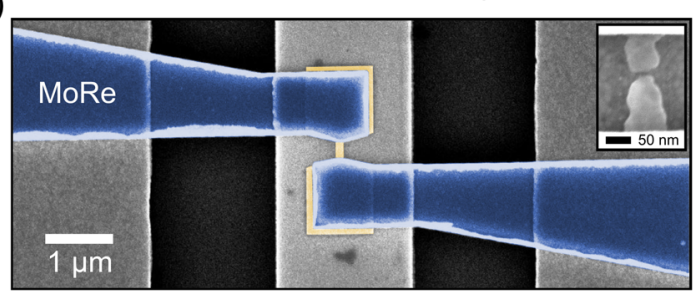

FIG. 1. Proximity induced interaction with an all organic radical molecule and device design. (a) Schematic representation of a radical molecule coupled to a proximity induced superconducting gold electrode. The red circle represents the unpaired spin at the center of the molecule. (b) False-colored scanning electron microscopy (SEM) image of a lithographically identical junction with superconducting electrodes colored blue and gold nanowire colored gold. The inset shows a SEM image of an electromigrated junction after measurement, scale bar $50 \mathrm{~nm}$.

coupled to intrinsically superconducting leads [36]. Gold is an attractive intermediary material because it allows coupling to a single molecule through a robust sulfur-gold bond leading to higher stability and stronger coupling.

Here we report on investigations of the large- $U$ regime of the superconductor-QD system and the observation of Shiba states in a completely proximitized superconducting junction hosting a model spin 1/2 impurity. As opposed to direct coupling to a bulk superconductor with a phase coherent condensate giving rise to spin induced Shiba states, Shiba states in our system are supported by correlated electron-hole (Andreev) pairs. Figure 1(a) depicts this situation in which a magnetic impurity (in our case a molecule with an unpaired spin at its center signified by a red circle, see Supplemental Material for details [38]) on the left side is tunnel coupled to a proximity-induced superconductor on the right side. The proximity effect in the gold is mediated by the well-known Andreev reflection process that occurs at the interface between the normal metal (gold region) and the superconductor (blue region) giving rise to Andreev pairs in the gold leads [40] which can interact with the molecule through an exchange coupling. As a result of a variable molecule-electrode coupling for different devices, we access both the singlet and doublet ground states (and their corresponding Shiba excited states) of the large- $U$ superconductor-QD phase diagram. We further corroborate these results through calculations based on the Anderson impurity model while taking into account the proximity induced nature of the gold electrodes.

Device design.-The junction fabrication and characterization have been detailed in Refs. [41,42] which we briefly summarize here. Figure 1(b) shows a false colored scanning electron microscopy (SEM) image of a representative junction. The blue colored electrodes are the bulk superconducting banks created by sputtering molybdenumrhenium $\left(60 / 40, T_{c} \approx 8.5 \mathrm{~K}\right)$. The gold colored nanowire and contact pads are created by electron-beam evaporation of gold (thickness, $12 \mathrm{~nm}$ ). The gray vertical strip under the junction is a $\mathrm{AlO}_{x} / \mathrm{AuPd}$ back gate which can be used to electrostatically gate the junction. A combination of electromigration and a self-breaking technique is used to open a nanogap in the gold nanowire. The inset shows a SEM image of a typical nanogap after electromigration.

Several empty gaps are measured at low temperatures in order to characterize the superconducting proximity effect in the gold electrodes without molecules (see Supplemental Material for details [43]. In order to carry out measurements on the polychlorotriphenylmethyl (PTM) molecule (see Supplemental Material for details [38]), a solution of molecules (concentration $1 \mathrm{mM}$ ) is drop cast onto an array of 24 junctions before electromigration. After the electromigration and self-breaking of all junctions, the solution is evaporated leaving behind roughly a monolayer of molecules and the sample is cooled down in a dilution refrigerator (base temperature $100 \mathrm{mK}$ ).

We find that the normal state characteristics (and Kondo effect) of the molecular junctions are consistent with previous reports of the same molecule in completely gold break junctions [46] (see Supplemental Material for details [47]. Furthermore, from the linear conductance in the Kondo regime we estimate an asymmetry in the couplings $\left(\Gamma_{L}, \Gamma_{R}\right)$ to the leads of $\Gamma_{L} / \Gamma_{R} \approx 5 \times 10^{-4}$.

The superconducting state.-Moving now to the characteristics in the superconducting state, the black curve in Fig. 2(a) shows the differential conductance as a function of voltage bias in the superconducting state $(B=0 \mathrm{~T})$ for device $D$. The red curve in the same panel is the measurement in the normal state $(B=200 \mathrm{mT})$ showing the zero bias peak arising from the Kondo effect discussed in the Supplemental Material [47]. In the superconducting state two peaks are discernible, symmetric in bias, accompanied by side dips at higher bias which are qualitatively distinctive from the empty junction curves shown in the Supplemental Material [43]. These peaks signal excitations of the coupled superconductor-QD system and have been shown to be associated with (multiple) Andreev reflections (MARs) and spin induced Yu-Shiba-Rusniov states. Conductance peaks due to MARs, however, are not accompanied by side dips and become less probable as the asymmetry between the left and right leads is increased [3,27]. Asymmetries of $\Gamma_{L} / \Gamma_{R} \approx$ $10^{-2}-10^{-3}$ in nanowire experiments are enough to completely suppress MAR contributions [3]. With the built-in high asymmetry of the couplings and given the spin $1 / 2$ Kondo effect in the normal state, a natural explanation for the excitation peaks in our hybrid molecular devices is that they originate from spin induced Shiba states.

Figure 2(c) shows a schematic of the coupling situation and density of states. The blue colored electrode and 
(a)

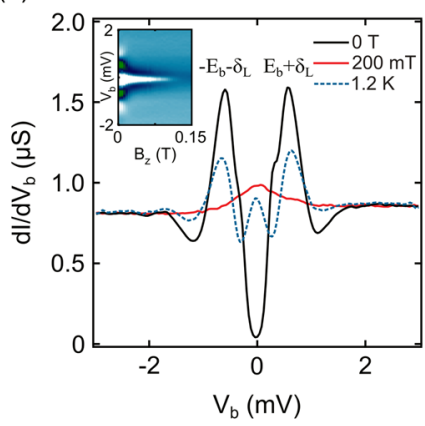

(b)

(c)

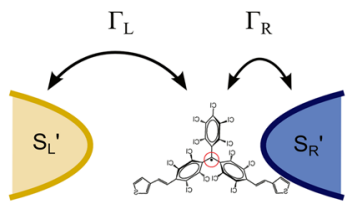

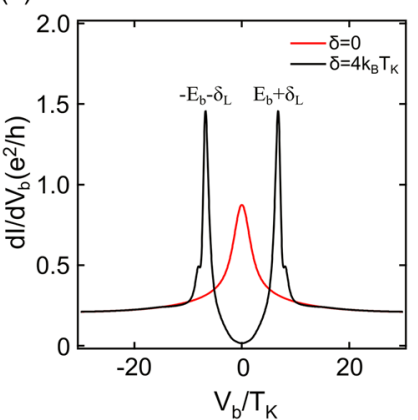

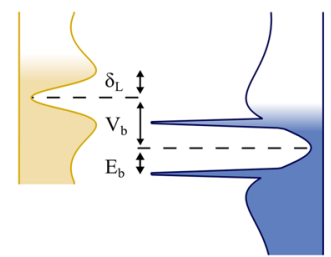

FIG. 2. Characterization and calculations of a molecular junction in the superconducting state. (a) Low temperature $(100 \mathrm{mK})$ measurement of the differential conductance $\left(d I / d V_{b}\right)$ as a function of voltage bias $\left(V_{b}\right)$ of a radical molecular junction at zero magnetic field (superconducting state) and $200 \mathrm{mT}$ (normal state). The blue dashed curve shows the same measurement at $1.2 \mathrm{~K}(B=0 \mathrm{~T})$. The inset shows the magnetic field dependence of the Shiba peaks. (b) Theoretical calculations of the modeled system taking into account the proximity-induced DOS of the leads. (c) Schematic representation of the probing of the Shiba excited state as a result of the coupling of the radical molecule with the proximity-induced superconducting $\mathrm{Au}$ electrode.

corresponding DOS represent the hybridization of the radical with the more strongly coupled electrode resulting in Shiba excited states. Microscopically, these states arise from the interaction of Andreev pairs at finite energy in the proximityinduced lead and the unpaired spin of the radical molecule. The weakly coupled gold lead on the left side probes the hybridization of the molecule with the right electrode. This results in conductance peaks in Fig. 2(a) at energies of $\pm\left(E_{b} \pm \delta_{L}\right)$ where $E_{b}$ is the excited state energy and $\delta_{L}$ is the proximity-induced gap of the probe electrode. In addition to these peaks, side shoulders are visible at lower bias voltage which we interpret as Shiba replicas. Shiba replicas are visible for a sufficient density of quasiparticles in the minigap of the probe electrode which is reasonable considering the soft proximity-induced gap of our empty junctions (see Supplemental Material [43]). With an increase in temperature we furthermore observe the emergence of an anomalous zero-bias peak [dashed curve in Fig. 2(a)] which we interpret as a mini Kondo due to increased quasiparticle filling (see Supplemental Material [50]).

A comparison of the characteristic energies $\left(\delta_{\text {avg }}\right.$ vs $k_{B} T_{K}$ ) for device $D$ allows us to determine the ground state of the coupled system. The edges of the dips in Fig. 2(a) roughly correspond to $\delta_{L}+\delta_{R}$ from which we calculate an average induced gap of $\delta_{\text {avg }}=\left(\delta_{L}+\delta_{R}\right) / 2=0.8 \mathrm{meV}$.

Compared with the Kondo energy extracted from the normal state temperature dependence of the Kondo peak $\left(k_{B} T_{K}=0.2 \mathrm{meV}\right)$ (see Supplemental Material [47]), we find that the average induced gap is 4 times larger. In this device, the larger finite Andreev pair energy results in a doublet ground state where the radical's spin is not sufficiently screened by quasiparticle states near the Fermi energy in the superconducting state to allow the Kondo singlet. Application of a perpendicular magnetic field, however, allows us to tune the ground state from the doublet to the Kondo singlet [see inset of Fig. 2(a)].

Our low temperature measurements are further supported by calculations. Starting with a modified Anderson impurity model (Anderson Hamiltonian coupled to leads modeled by BCS Hamiltonians) we calculate the differential conductance as a function of bias voltage using the noncrossing approximation [3,51,52] (see Supplemental Material for details [53]). The broadening of the DOS due to the proximity effect is handled by introducing the Dynes function which is taken as a phenomenological broadening term that softens the BCS DOS of the bulk reservoirs $[3,62]$. Figure 2(b) shows a conductance curve from this calculation where the main features of the experimental curve can be reproduced. A zero-bias peak is present when the order parameter in the leads is set to zero (red curve). In the superconducting state with $\delta=4 k_{B} T_{K}$ (for calculations $\left.\delta_{L}=\delta_{R}=\delta\right)$ and broadening $(0.15 \delta)$, two peaks can be seen with accompanying side dips at higher bias (black curve). Note that we have also incorporated an asymmetry in the lead couplings of $\Gamma_{L} / \Gamma_{R}=1 / 2$ to better model the results. Quantitative differences are primarily due to the exact shape of the DOS for each gold lead. Differences in the lengths of the two gold leads (see Supplemental Material for details [43]) mean the broadening for the two leads would be different $\left(\delta_{L} \neq \delta_{R}\right)$. Additionally, the shape of the gold lead near the molecular junction ultimately plays a role in the proximity effect and thus the shape of the DOS which would require more intensive calculations.

Ground state spectroscopy in the superconducting state.-Depending on the relative energies of the proximity-induced gap and the Kondo energy, the ground state of the system, even in the superconducting state, can take the form of a doublet for weak coupling $\left(k_{B} T_{K}<\delta_{\text {avg }}\right)$ or a Kondolike singlet for strong coupling $\left(k_{B} T_{K}>\delta_{\text {avg }}\right)$. By virtue of a variable molecule-electrode coupling, we probe a large range of Kondo energies relative to the average induced gap energy. In the Supplemental Material [63] we show an overview of the 7 devices $(A-G)$ having similar characteristics ordered by increasing Kondo energy. The Kondo temperature ranges from $(\approx 1 \mathrm{~K}$ to $18 \mathrm{~K})$ for the seven devices.

In Fig. 3 we show the weakest and strongest Kondo energy devices (junctions $A$ and $G$ ). For junction $A$ a Kondo peak cannot be discerned in the normal state [red curve Fig. 3(a)] but a splitting $(g=2.1)$ of the background is observed at higher magnetic fields [Fig. 3(b)]. We estimate 

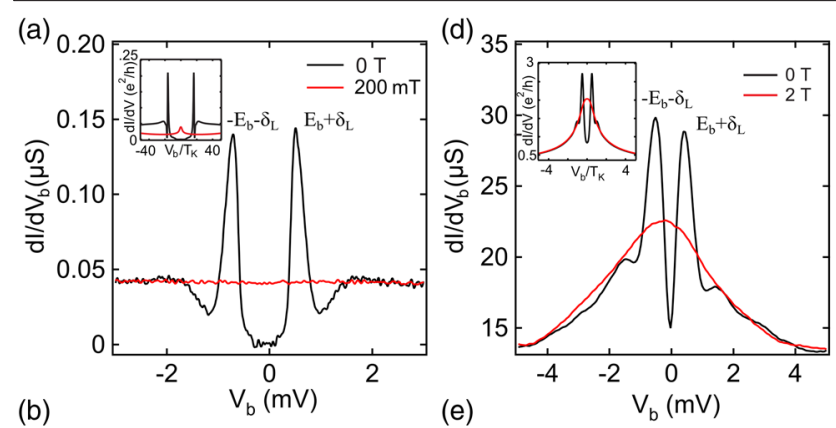

(b)
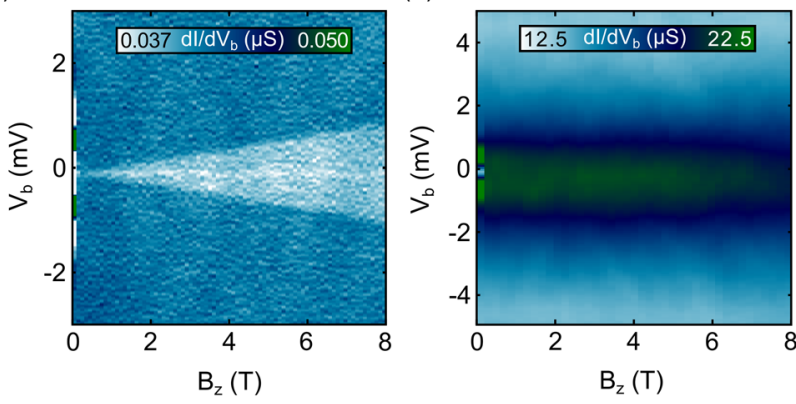

(c)

(f)
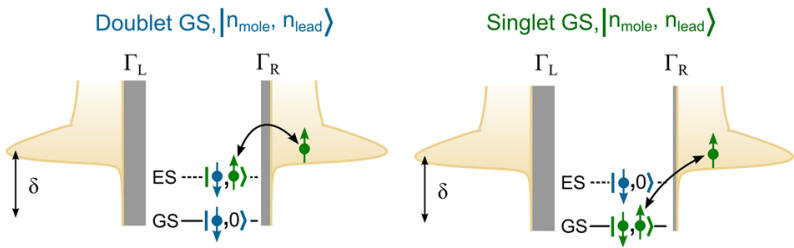

FIG. 3. Ground state spectroscopy of the hybrid radical system. (a) The differential conductance $\left(d I / d V_{b}\right)$ as a function of voltage bias $\left(V_{b}\right)$ of a radical molecular junction at zero magnetic field (superconducting state) and $200 \mathrm{mT}$ (normal state) in the doublet ground state regime $\left(k_{B} T_{K}<\delta_{\text {avg }}\right)$. Inset shows calculations for this regime $\left(\delta=9 k_{B} T_{K}\right)$. (b) Color plot of the differential conductance $\left(d I / d V_{b}\right)$ as a function of voltage bias and magnetic field for the junction in panel (a). (c) Excitation picture of the ground and excited states in the doublet ground state regime. (d) The differential conductance $\left(d I / d V_{b}\right)$ as a function of voltage bias $\left(V_{b}\right)$ of a radical molecular junction at zero magnetic field (superconducting state) and $2 \mathrm{~T}$ (normal state) in the singlet ground state regime $\left(k_{B} T_{K}>\delta_{\text {avg }}\right)$. Inset shows calculations for this regime $\left(\delta=0.5 k_{B} T_{K}\right)$. (e) Color plot of the differential conductance $\left(d I / d V_{b}\right)$ as a function of voltage bias and magnetic field for the junction in panel (d). (f) Excitation picture of the ground and excited states in the singlet ground state regime.

a Kondo temperature of $\approx 1 \mathrm{~K}$ from the critical field $\left[B_{c} \approx 0.5 k_{B} T_{K} /\left(g \mu_{B}\right)\right]$ at which the background begins to split in Fig. 3(b) $\left(B_{c} \approx 0.36 \mathrm{~T}\right)$. Following the analysis for device $D$ above, we estimate an average induced gap of $0.8 \mathrm{meV}$. Comparing the two energy scales $(0.8 \mathrm{meV}$ vs $0.09 \mathrm{meV}$ ), this device is similar to device $D$ in which the doublet ground state wins over the Kondo singlet. The inset of Fig. 3(a) shows the calculations for this regime where we have taken $\delta=9 k_{B} T_{K}$ and built in an asymmetry of $\Gamma_{R} / \Gamma_{L}=20$ to better simulate the results. Shiba peaks in this regime correspond to singlet excitations of the doublet ground state. This situation is depicted in Fig. 3(c) in the excitation picture where a thick barrier and reduced quasiparticle states near the Fermi energy prevent the Kondo singlet from claiming the ground state of the coupled system. Here we signify the ground state and the excited state by occupancies of the molecule and the leads, $\left|n_{\text {mole }}, n_{\text {lead }}\right\rangle$. The Shiba excited state for this ground state, $|\downarrow, \uparrow\rangle$, is a singlet composed of the molecule's unpaired spin and an electron from a correlated Andreev pair in the leads (signified by the black arrow).

In Fig. 3(d) we present the device with the largest Kondo temperature observed ( $18 \mathrm{~K}$, estimated from the peak width). Comparing the characteristic energies for this device $\left(\delta_{\text {avg }}=0.7 \mathrm{meV}, k_{B} T_{K}=1.6 \mathrm{meV}\right)$ we find that the large Kondo energy allows for screening of the radical's spin even in the superconducting state. This results in a singlet ground state at $B=0 \mathrm{~T}$. Calculations for this regime are shown in the inset of Fig. 3(d) where we have taken $\delta=0.5 k_{B} T_{K}$ and a broadening of $0.25 \delta$. Excitations of this ground state are the magnetic doublet. This is depicted in Fig. 3(f) in the excitation picture. A weak barrier allows for screening from electrons in the proximity-induced leads and a singlet state is formed by the combination of the radical's spin and an electron in the leads.

Finally, using the average proximity induced gap, we make a qualitative estimate of the bound state energies for the 7 devices and extract the approximate ratios of $k_{B} T_{K} / \delta_{\text {avg }}$ for which a quantum phase transition occurs from the doublet ground state to the singlet ground state (see the Supplemental Material for details [64]). Of the seven devices studied, four were found to have induced superconducting gaps with energies greater than their respective Kondo energy, and three were found to have energies less than their Kondo energy. Taken all together, the data suggest that a quantum phase transition occurs for $k_{B} T_{K} / \delta_{\text {avg }}$ between 0.9 and 1.1. This is in agreement with STM studies of molecules on a $\mathrm{Pb}$ surface $\left(k_{B} T_{K} / \Delta \approx 1\right)$ [2] but slightly higher than early numerical renormalization group (NRG) theory calculations which predict a transition at $k_{B} T_{K} / \Delta \approx 0.3$ (Ref. [65]) and more recent investigations in nanowire systems showing quantitative agreement between experiment and NRG calculations for a phase transition occurring at $k_{B} T_{K} / \Delta \approx 0.6$ (Ref. [66]).

Conclusion.-In conclusion, we have presented an investigation of the large- $U$ superconductor-QD phase diagram in the form of a radical molecule coupled to a proximity induced superconductor. In the superconducting state, we observe excitations which are characteristic of Shiba states as a result of the coupling between the radical and a proximity-induced superconductor. By applying a finite magnetic field, the proximity effect can be suppressed which allows a spin $1 / 2$ Kondo effect. For the devices with the weakest and strongest Kondo energies we are able to probe both the doublet and singlet ground states which give rise to the singlet and doublet Shiba excited states, respectively. Finally, we estimate that the quantum phase transition 
from the doublet to the singlet ground state occurs for ratios of $k_{B} T_{K}$ to $\delta_{\text {avg }}$ from 0.9 to 1.1. These findings are supported by calculations of the Anderson impurity model modified to account for the proximity-induced superconducting electrodes. Hybrid molecular junctions offer a unique investigation of the superconductor-QD system. In particular, with a suitable choice of molecule, an in-situ tunable Kondo effect would allow direct driving of the singlet to doublet quantum phase transition for a spin $1 / 2$ impurity and extraction of the energies at which it occurs $[2,65,67]$.

The authors thank Jens Paaske for insightful discussions. The authors acknowledge financial support by the Dutch Organization for Fundamental research (FOM), the Ministry of Education, Culture, and Science (OCW), the Netherlands Organization for Scientific Research (NWO), and an ERC advanced grant (MolS@MolS). C.F., M.M.-T., C. R., and J. V. acknowledge the financial support from Spanish Ministry of Economy and Competitiveness, through the DGI (CTQ2013-40480-R) and Severo Ochoa Programme for Centres of Excellence in R\&D (SEV-2015-0496), the Generalitat de Catalunya (2014SGR-17) and the Networking Research Center of Bioengineering, Biomaterials and Nanomedicine (CIBER-BBN). T. M. K. acknowledges the support from the European Research Council Advanced Grant No. 339306 (METIQUM) and from the Ministry of Education and Science of the Russian Federation, Contract No. 14.B25.31.0007. R. A. acknowledges Grants No. FIS2012-33521 and No. FIS-2015-64654 (MINECO/FEDER).

*jisland@physics.ucsb.edu

Present address: Department of Physics, University of California, Santa Barbara CA 93106 USA.

[1] S. De Franceschi, L. Kouwenhoven, C. Schönenberger, and W. Wernsdorfer, Hybrid superconductor-quantum dot devices, Nat. Nanotechnol. 5, 703 (2010).

[2] K. J. Franke, G. Schulze, and J. I. Pascual, Competition of superconducting phenomena and kondo screening at the nanoscale, Science 332, 940 (2011).

[3] E. J. H. Lee, X. Jiang, R. Aguado, G. Katsaros, C. M Lieber, and S. De Franceschi, Zero-Bias Anomaly in a Nanowire Quantum Dot Coupled to Superconductors, Phys. Rev. Lett. 109, 186802 (2012).

[4] W. Chang, V. E. Manucharyan, T. S. Jespersen, J. Nygård, and C. M. Marcus, Tunneling Spectroscopy of Quasiparticle Bound States in a Spinful Josephson Junction, Phys. Rev. Lett. 110, 217005 (2013).

[5] E J. H. Lee, X. Jiang, M. Houzet, R. Aguado, Charles M Lieber, and S. De Franceschi, Spin-resolved Andreev levels and parity crossings in hybrid superconductor-semiconductor nanostructures, Nat. Nanotechnol. 9, 79 (2014).

[6] R. Žitko, J. S. Lim, J. S. Lim, R. López, and R. Aguado, Shiba states and zero-bias anomalies in the hybrid normalsuperconductor anderson model, Phys. Rev. B 91, 045441 (2015).
[7] N. Hatter, B. W Heinrich, M. Ruby, J. I Pascual, and K. J Franke, Magnetic anisotropy in shiba bound states across a quantum phase transition, Nat. Commun. 6, 8988 (2015).

[8] M. Źonda, V. Pokorný, V. Janiš, and T. Novotný, Perturbation theory for an anderson quantum dot asymmetrically attached to two superconducting leads, Phys. Rev. B 93, 024523 (2016).

[9] M. Ruby, F. Pientka, Y. Peng, F. von Oppen, B. W Heinrich, and K. J. Franke, Tunneling Processes into Localized Subgap States in Superconductors, Phys. Rev. Lett. 115, 087001 (2015).

[10] J. Pillet, C. Quay, P. Morfin, C. Bena, A. L. Yeyati, and P. Joyez, Andreev bound states in supercurrent-carrying carbon nanotubes revealed, Nat. Phys. 6, 965 (2010).

[11] J.-D. Pillet, P. Joyez, R. Žitko, and M. F. Goffman, Tunneling spectroscopy of a single quantum dot coupled to a superconductor: From kondo ridge to andreev bound states, Phys. Rev. B 88, 045101 (2013).

[12] V. Mourik, K. Zuo, S. M. Frolov, S. R. Plissard, E. P. A. M. Bakkers, and L. P. Kouwenhoven, Signatures of majorana fermions in hybrid superconductor-semiconductor nanowire devices, Science 336, 1003 (2012).

[13] W. Chang, S. M. Albrecht, T. S. Jespersen, F. Kuemmeth, P. Krogstrup, J. Nygård, and C. M. Marcus, Hard gap in epitaxial semiconductor-superconductor nanowires, Nat. Nanotechnol. 10, 232 (2015).

[14] T. Meng, S. Florens, and P. Simon, Self-consistent description of Andreev bound states in josephson quantum dot devices, Phys. Rev. B 79, 224521 (2009).

[15] L Yu, Bound state in superconductors with paramagnetic impurities, Acta Phys. Sin. 21, 75 (1965).

[16] H. Shiba, Classical spins in superconductors, Prog. Theor. Phys. 40, 435 (1968).

[17] A. I. Rusinov, Superconductivity near a paramagnetic impurity, JETP Lett. 9, 85 (1969).

[18] A. V Balatsky, I. Vekhter, and J.-X. Zhu, Impurity-induced states in conventional and unconventional superconductors, Rev. Mod. Phys. 78, 373 (2006).

[19] A. Yazdani, B. A. Jones, C. P. Lutz, M. F. Crommie, and D. M. Eigler, Probing the local effects of magnetic impurities on superconductivity, Science 275, 1767 (1997).

[20] S. Nadj-Perge, I. K. Drozdov, B. A. Bernevig, and A. Yazdani, Proposal for realizing majorana fermions in chains of magnetic atoms on a superconductor, Phys. Rev. B 88, 020407 (2013).

[21] F. Pientka, L. I. Glazman, and F. von Oppen, Topological superconducting phase in helical shiba chains, Phys. Rev. B 88, 155420 (2013).

[22] S. Nadj-Perge, I. K. Drozdov, J. Li, H. Chen, S. Jeon, J. Seo, A. H. MacDonald, B. A. Bernevig, and A. Yazdani, Observation of majorana fermions in ferromagnetic atomic chains on a superconductor, Science 346, 602 (2014).

[23] L. Dumoulin, E. Guyon, and P. Nedellec, Tunneling study of localized bands in superconductors with magnetic impurities (normal kondo alloys in the superconducting proximity), Phys. Rev. B 16, 1086 (1977).

[24] K. Machida, Proximity effect for superconductors containing transition metal impurities. I, J. Low Temp. Phys. 27, 737 (1977). 
[25] K. Machida and L. Dumoulin, Proximity effect for superconductors containing transition metal impurities. II, J. Low Temp. Phys. 31, 143 (1978).

[26] G. C. Ménard et al., Coherent long-range magnetic bound states in a superconductor, Nat. Phys. 11, 1013 (2015).

[27] B. M. Andersen, K. Flensberg, V. Koerting, and J. Paaske, Nonequilibrium Transport Through a Spinful Quantum Dot with Superconducting Leads, Phys. Rev. Lett. 107, 256802 (2011).

[28] B.-K. Kim, Y.-H. Ahn, J.-J. Kim, M.-S. Choi, M.-H. Bae, K. Kang, J. S. Lim, R. López, and N. Kim, Transport Measurement of Andreev Bound States in a Kondo-Correlated Quantum Dot, Phys. Rev. Lett. 110, 076803 (2013).

[29] A. Kumar, M. Gaim, D. Steininger, A. Levy Yeyati, A. Martín-Rodero, A. K. Hüttel, and C. Strunk, Temperature dependence of andreev spectra in a superconducting carbon nanotube quantum dot, Phys. Rev. B 89, 075428 (2014).

[30] A. Yu Kitaev, Unpaired majorana fermions in quantum wires, Phys. Usp. 44, 131 (2001).

[31] J. Alicea, Majorana fermions in a tunable semiconductor device, Phys. Rev. B 81, 125318 (2010).

[32] J. D. Sau, R. M. Lutchyn, S. Tewari, and S. D. Sarma, Generic New Platform for Topological Quantum Computation using Semiconductor Heterostructures, Phys. Rev. Lett. 104, 040502 (2010).

[33] Y. Oreg, G. Refael, and F. von Oppen, Helical Liquids and Majorana Bound States in Quantum Wires, Phys. Rev. Lett. 105, 177002 (2010).

[34] J. Alicea, New directions in the pursuit of majorana fermions in solid state systems, Rep. Prog. Phys. 75, 076501 (2012).

[35] S. M. Albrecht, A. P. Higginbotham, M. Madsen, F. Kuemmeth, T. S. Jespersen, J. Nygård, P Krogstrup, and C. M. Marcus, Exponential protection of zero modes in majorana islands, Nature (London) 531, 206 (2016).

[36] C. B. Winkelmann, N. Roch, W. Wernsdorfer, V. Bouchiat, and F. Balestro, Superconductivity in a single-c60 transistor, Nat. Phys. 5, 876 (2009).

[37] K. Luo and Z. Yao, Fabrication of nanometer-spaced superconducting pb electrodes, Appl. Phys. Lett. 95, 113115 (2009).

[38] See Supplemental Material at http://link.aps.org/ supplemental/10.1103/PhysRevLett.118.117001 for a complete description of the molecule which includes Ref. [39].

[39] M. Ballester, Inert free radicals (IFR): A unique trivalent carbon species, Acc. Chem. Res. 18, 380 (1985).

[40] A. F. Andreev, The thermal conductivity of the intermediate state in superconductors, Sov. Phys. JETP 19, 1228 (1964).

[41] R. Gaudenzi, J. O. Island, J. De Bruijckere, E. Burzurí, T. M. Klapwijk, and H. S. J. Van der Zant, Superconducting molybdenum-rhenium electrodes for single-molecule transport studies, Appl. Phys. Lett. 106, 222602 (2015).

[42] K. ONeill, E. A. Osorio, and H.S.J. Van der Zant, Self-breaking in planar few-atom au constrictions for nanometer-spaced electrodes, Appl. Phys. Lett. 90, 133109 (2007).

[43] See Supplemental Material at http://link.aps.org/ supplemental/10.1103/PhysRevLett.118.117001 for low temperature characteristics of an empty gap, which includes Refs. [44,45].
[44] M. Wolz, C. Debuschewitz, W. Belzig, and E. Scheer, Evidence for attractive pair interaction in diffusive gold films deduced from studies of the superconducting proximity effect with aluminum, Phys. Rev. B 84, 104516 (2011).

[45] W. Belzig, C. Bruder, and G. Schön, Local density of states in a dirty normal metal connected to a superconductor, Phys. Rev. B 54, 9443 (1996).

[46] R. Frisenda, R. Gaudenzi, C. Franco, M. Mas-Torrent, C. Rovira, J. Veciana, I. Alcon, S. T. Bromley, E. Burzurí, and H. S. J Van der Zant, Kondo effect in a neutral and stable all organic radical single molecule break junction, Nano Lett. 15, 3109 (2015).

[47] See Supplemental Material at http://link.aps.org/ supplemental/10.1103/PhysRevLett.118.117001 for normal state characterization of a molecular junction which includes Refs. [48,49].

[48] J. J. Parks, A. R. Champagne, G. R. Hutchison, S. FloresTorres, H. D. Abruna, and D. C. Ralph, Tuning the Kondo Effect with a Mechanically Controllable Break Junction, Phys. Rev. Lett. 99, 026601 (2007).

[49] T. A. Costi, A. C. Hewson, and V. Zlatic, Transport coefficients of the anderson model via the numerical renormalization group, J. Phys. Condens. Matter 6, 2519 (1994).

[50] See Supplemental Material at http://link.aps.org/ supplemental/10.1103/PhysRevLett.118.117001 for temperature dependence of the mini Kondo along with model calculations

[51] A. A. Clerk and V. Ambegaokar, Loss of $\pi$-junction behavior in an interacting impurity josephson junction, Phys. Rev. B 61, 9109 (2000).

[52] G. Sellier, T. Kopp, J. Kroha, and Y. S Barash, $\pi$ junction behavior and andreev bound states in kondo quantum dots with superconducting leads, Phys. Rev. B 72, 174502 (2005).

[53] See Supplemental Material at http://link.aps.org/ supplemental/10.1103/PhysRevLett.118.117001 for a complete description of the theoretical model which includes Refs. [54-61].

[54] D. C. Langreth and P. Nordlander, Derivation of a master equation for charge-transfer processes in atom-surface collisions, Phys. Rev. B 43, 2541 (1991).

[55] N. S. Wingreen and Y. Meir, Anderson model out of equilibrium: Noncrossing-approximation approach to transport through a quantum dot, Phys. Rev. B 49, 11040 (1994).

[56] M H. Hettler, J. Kroha, and S. Hershfield, Nonlinear Conductance for the Two Channel Anderson Model, Phys. Rev. Lett. 73, 1967 (1994).

[57] M. H. Hettler, J. Kroha, and S. Hershfield, Nonequilibrium dynamics of the anderson impurity model, Phys. Rev. B 58, 5649 (1998).

[58] R. Aguado and D. C. Langreth, Kondo effect in coupled quantum dots: A noncrossing approximation study, Phys. Rev. B 67, 245307 (2003).

[59] T. A. Costi, J. Kroha, and P. Wölfle, Spectral properties of the anderson impurity model: Comparison of numericalrenormalization-group and noncrossing-approximation results, Phys. Rev. B 53, 1850 (1996).

[60] J. Devreeese, Linear and Nonlinear Electron Transport in Solids, Vol. 17 (Springer, New York, 1976). 
[61] Y. Meir and N. S. Wingreen, Landauer Formula for the Current through an Interacting Electron Region, Phys. Rev. Lett. 68, 2512 (1992).

[62] R. C. Dynes, V. Narayanamurti, and J P. Garno, Direct Measurement of Quasiparticle-Lifetime Broadening in a Strong-Coupled Superconductor, Phys. Rev. Lett. 41, 1509 (1978).

[63] See Supplemental Material at http://link.aps.org/ supplemental/10.1103/PhysRevLett.118.117001 for an overview of the seven molecular devices having similar characteristics.

[64] See Supplemental Material at http://link.aps.org/ supplemental/10.1103/PhysRevLett.118.117001 for a plot of the Shiba energy $\left(E_{b}\right)$ versus the Kondo energy $\left(k_{B} T_{K}\right)$ for all seven molecular devices.

[65] O. Sakai, Y. Shimizu, H. Shiba, and K. Satori, Numerical renormalization group study of magnetic impurities in superconductors. ii. dynamical excitation spectra and spatial variation of the order parameter, J. Phys. Soc. Jpn. 62, 3181 (1993).

[66] E. J. H. Lee, X. Jiang, R. Zitko, R. Aguado, C. M. Lieber, and S. De Franceschi, Scaling of sub-gap excitations in a superconductor-semiconductor nanowire quantum dot, arXiv: 1609.07582.

[67] T. Matsuura, The effects of impurities on superconductors with kondo effect, Prog. Theor. Phys. 57, 1823 (1977). 